\title{
A combined Compton and coded-aperture telescope for medium-energy gamma-ray astrophysics
}

\author{
Michelle Galloway $^{1}$, Andreas Zoglauer ${ }^{2}$, Steven E. Boggs ${ }^{2}$, and Mark Amman ${ }^{3}$ \\ ${ }^{1}$ Universität Zürich, Physik-Institut, Winterthurerstrasse 190, Zürich 8057, Switzerland \\ e-mail: galloway@physik.uzh.ch \\ ${ }^{2}$ University of California at Berkeley, Space Sciences Laboratory, 7 Gauss Way, Berkeley, CA 94720, USA \\ ${ }^{3}$ Lawrence Berkeley National Laboratory, 1 Cyclotron Road, Berkeley, CA 94720, USA
}

Received 7 May 2017 / Accepted 31 January 2018

\begin{abstract}
A future mission in medium-energy gamma-ray astrophysics would allow for many scientific advancements, such as a possible explanation for the excess positron emission from the Galactic center, a better understanding of nucleosynthesis and explosion mechanisms in Type Ia supernovae, and a look at the physical forces at play in compact objects such as black holes and neutron stars. Additionally, further observation in this energy regime would significantly extend the search parameter space for low-mass dark matter. In order to achieve these objectives, an instrument with good energy resolution, good angular resolution, and high sensitivity is required. In this paper we present the design and simulation of a Compton telescope consisting of cubic-centimeter cadmium zinc telluride detectors as absorbers behind a silicon tracker with the addition of a passive coded mask. The goal of the design was to create a very sensitive instrument that is capable of high angular resolution. The simulated telescope achieved energy resolutions of $1.68 \%$ FWHM at $511 \mathrm{keV}$ and $1.11 \%$ at $1809 \mathrm{keV}$, on-axis angular resolutions in Compton mode of $2.63^{\circ} \mathrm{FWHM}$ at $511 \mathrm{keV}$ and $1.30^{\circ} \mathrm{FWHM}$ at $1809 \mathrm{keV}$, and is capable of resolving sources to at least $0.2^{\circ}$ at lower energies with the use of the coded mask. An initial assessment of the instrument in Compton-imaging mode yields an effective area of $183 \mathrm{~cm}^{2}$ at $511 \mathrm{keV}$ and an anticipated all-sky sensitivity of $3.6 \times 10^{-6}$ photons $\mathrm{cm}^{-2} \mathrm{~s}^{-1}$ for a broadened $511 \mathrm{keV}$ source over a two-year observation time. Additionally, combining a coded mask with a Compton imager to improve point-source localization for positron detection has been demonstrated.
\end{abstract}

Key words. gamma rays: general - techniques: high angular resolution - telescopes - instrumentation: detectors methods: observational - techniques: image processing

\section{Introduction}

The highly penetrative nature of gamma rays allows for a unique probe into the most violent explosions and dynamic sources in the Universe. As they travel through the Galaxy relatively undeterred, detected photons in the $\mathrm{MeV}$ to $\mathrm{GeV}$ regime directly point back to their cosmic source. Obtaining spatial, spectral, and temporal information at these wavelengths will help to decipher the physical mechanisms behind astrophysical sources and events and to distinguish between different theoretical models, and it may reveal new sources and phenomena. Particularly, imaging in this regime provides valuable information regarding the type, morphology, and spatial distribution of gamma-ray sources.

Previous missions using imaging telescopes such as COMPTEL (Schoenfelder et al. 1993) and the Compton Spectrometer and Imager (COSI) instrument (Kierans et al. 2017) have demonstrated good Compton-imaging capabilities in the medium-energy gamma-ray regime, for example, the all-sky map of ${ }^{26} \mathrm{Al}$ by COMPTEL (Oberlack et al. 1996) and the image of the Crab Nebula by the Nuclear Compton Telescope (Bandstra et al. 2011). Additionally, coded-aperture imagers such as the SPI (SPectrometer on INTEGRAL) on board the INTErnational Gamma-Ray Astrophysics Laboratory (INTEGRAL) spacecraft (Vedrenne et al. 2003) have provided images of the Galactic plane and a Galactic all-sky map of the $511 \mathrm{keV}$ positron annihilation line (Bouchet et al. 2010).
A next-generation telescope in the medium-energy gammaray regime requires good energy resolution for the study of line emissions resulting from Galactic nucleosynthesis. Good angular resolution is needed to resolve point sources, thus providing a means to correlate gamma-ray emission with sources that are seen in other wavebands. Additionally, a telescope must have good efficiency and a high sensitivity to detect and image sources above a complex background. The high sensitivity needed for an astrophysical instrument can be achieved through the capabilities of a Compton telescope with regard to its background-rejection capabilities and as a result of excellent energy, position, and timing resolution. Furthermore, adding a coded aperture to a Compton telescope can significantly improve its angular resolution to well below the Doppler limit (Zoglauer \& Kanbach 2003) within its masked field of view (FoV). Codedaperture Compton telescopes (CACTs) have previously been investigated with the Imager on Board the Integral Satellite (IBIS; Forot et al. 2007).

This paper describes a preliminary study of a Compton imager combined with a passive coded mask with the goal of achieving high sensitivity as well as the ability to resolve point sources in crowded fields such as the Galactic center (GC) region. The Compton imager uses cubic-centimeter cadmium zinc telluride (CdZnTe) detectors as absorber planes behind an array of double-sided silicon strip detectors (DSSD Si) as particle tracker. Large-volume CdZnTe detectors are an attractive candidate for a future instrument because of their good 
absorption, simple design, and minimal or no cooling requirements. A passive mask covering a narrow $\left(10^{\circ}\right) \mathrm{FoV}$ is added to the Compton telescope in order to improve the on-axis angular resolution for dedicated observations.

Through simulations of sources in the energy range between $200 \mathrm{keV}$ and $6 \mathrm{MeV}$ with particular emphasis on characterizing the instrument at $511 \mathrm{keV}$ (positron annihilation line) as well as at $1809 \mathrm{keV}\left({ }^{26} \mathrm{Al}\right.$ line $)$, an initial assessment of the achievable energy resolution, angular resolution, and effective area is given. The sensitivity for line sources and a continuum source is estimated based upon the expected background in a low-Earth orbit. Additionally, localization of two $511 \mathrm{keV}$ point sources separated by $0.2^{\circ}$ is demonstrated using combined Compton and coded-aperture imaging modes.

\section{Scientific motivation}

The $\mathrm{MeV}$ to $\mathrm{GeV}$ energy range remains largely unexplored since the success of COMPTEL more than two decades ago, yet many open questions remain that can only be fully answered through observations in this regime. For example, an excess of gamma rays in both the extragalactic (in the $\sim 0.2-100 \mathrm{MeV}$ ) regime (Ajello et al. 2009) and in the GC, the latter particularly from positrons (Prantzos et al. 2011), has consistently been observed over many decades without clear indications of their origins. The GC excess has no distinguishable counterparts at other wavelengths, and it is not yet determined whether the emission is diffuse or originates from point sources. Several candidates have been proposed as explanation, such as cosmic rays, low-mass dark matter, or millisecond pulsars. In the case of the extragalactic $\mathrm{MeV}$ background, its observed intensity cannot be accounted for by any known source population or emission mechanism. Future observations in the $\mathrm{MeV}$ domain, particularly with high angular resolution, would help to distinguish between diffuse emission candidates and point sources of these excesses. Spectral information and determination of the redshift and luminosity functions would provide additional clarification on the nature of the $\mathrm{MeV}$ background. Furthermore, pointed observations of the GC region or dwarf spheroidal galaxies in this energy regime, for instance, will significantly widen the search for annihilation products from $\mathrm{MeV}$ to sub-GeV dark matter, thus providing the capability to either detect a signature or set more stringent constraints on existing models (Boddy \& Kumar 2015).

Another area where exploring the $\mathrm{MeV}$ domain can yield significant advances is in the understanding of compact objects, such as active galactic nuclei (AGN), core-collapse and thermonuclear supernovae, and neutron stars. For relativistic jets and outflows from AGNs, the transition between the low-energy (X-ray) continuum to the spectral shapes observed in the $\mathrm{GeV}$ to $\mathrm{TeV}$ range occurs in the $\mathrm{MeV}$ regime. Therefore this gap holds a wealth of information regarding the acceleration processes that occur within and around AGNs (Padovani et al. 2017). For Type 1a supernovae, spectral and luminosity measurements in the $\mathrm{MeV}$ range can provide important information about their evolution with respect to look-back time and metallicity; such an understanding is a prerequisite for using them as standard candles in precision cosmology (Phillips 1993). With respect to neutron stars, a recent example of the relevance of gamma-ray astronomy at the dawn of a multi-messenger era is the detection of the gravitational wave GW170817 in temporal coincidence with the gamma-ray burst GRB 170817A (Abbott et al. 2017). Although it was long suspected that a neutron star merger could be a progenitor of a short-duration GRB, confirmation was only possible through such a coincident measurement.

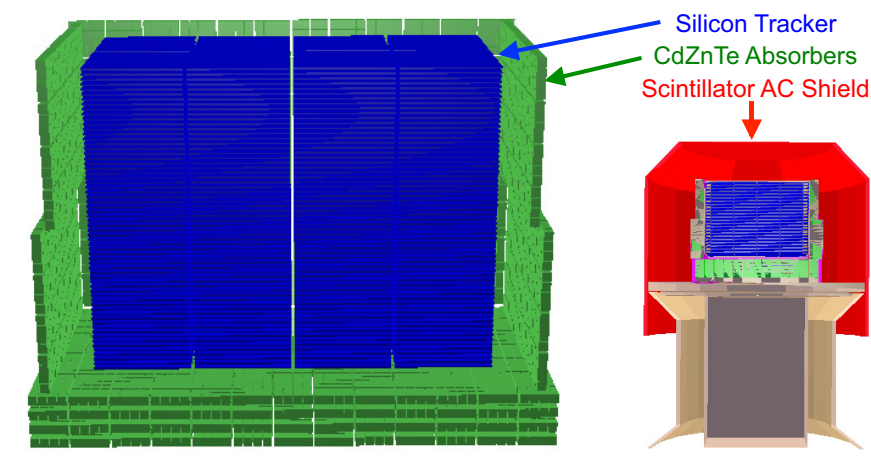

Fig. 1. Left: mass model of Compton telescope consisting of a silicon tracker array (blue) and CdZnTe absorber planes (green). Right: mass model of the organic scintillator anti-coincidence (AC) shield surrounding tracker and absorber detectors.

Because additional information in both the prompt and afterglow emissions from GRBs is encoded in the MeV domain, future detection and imaging in this regime will contribute to our understanding of the properties, environment, and evolution of GRB sources and will also provide the multi-messenger community with a relatively instantaneous localization of progenitor systems.

\section{Telescope design}

The proposed design of the telescope is shown in Fig. 1. The Compton telescope, left, consists of a silicon tracker (blue) in which the first Compton interaction takes place and a CdZnTe absorber (green) surrounding the tracker on five sides. Figure 1, right, shows the Compton telescope mounted on a support structure and surrounded by an organic plastic scintillator dome (red). The dome serves as an anti-coincidence (AC) shield to veto interactions of charged particles. An electronics box is contained within the pedestal of the support structure. The passive coded mask comprised of tungsten pixels above the tracker is shown in Fig. 2. Additional passive materials such as front-end electronics for all detectors, housing for the CdZnTe detectors, and a support frame for the tracker are included in the model. The total mass of the proposed instrument with support structure and shielding is $\sim 530 \mathrm{~kg}$. This mass is feasible for a Mid-sized Explorer (MIDEX) NASA mission.

\subsection{Silicon tracker}

The Compton tracker consists of multiple layers of double-sided silicon strip detectors and is based upon the Gamma-Ray burst Investigation via Polarimetry and Spectroscopy (GRIPS) telescope (Greiner et al. 2009; Zoglauer et al. 2008). A low-Z material such as silicon $(Z=14)$ is a good choice for a Compton scatter plane, as an incident photon can scatter many times in the tracker before becoming fully absorbed. These multiple interactions allow for a higher probability of correct Compton reconstruction, thus improved background rejection. Additionally, low- $Z$ materials such as silicon have a smaller contribution from Doppler broadening to the angular resolution as well as a higher Compton cross-section (relative to its photoabsorption cross-section) than medium- $Z$ to high- $Z$ materials such as $\mathrm{Ge}$ and CdZnTe. Simulated detector parameters include low-energy and trigger thresholds of $5 \mathrm{keV}$ and $10 \mathrm{keV}$, respectively, and a $1 \sigma$ FWHM energy resolution over a $0.2-10 \mathrm{MeV}$ energy range. This allows for a telescope with good overall energy resolution. 


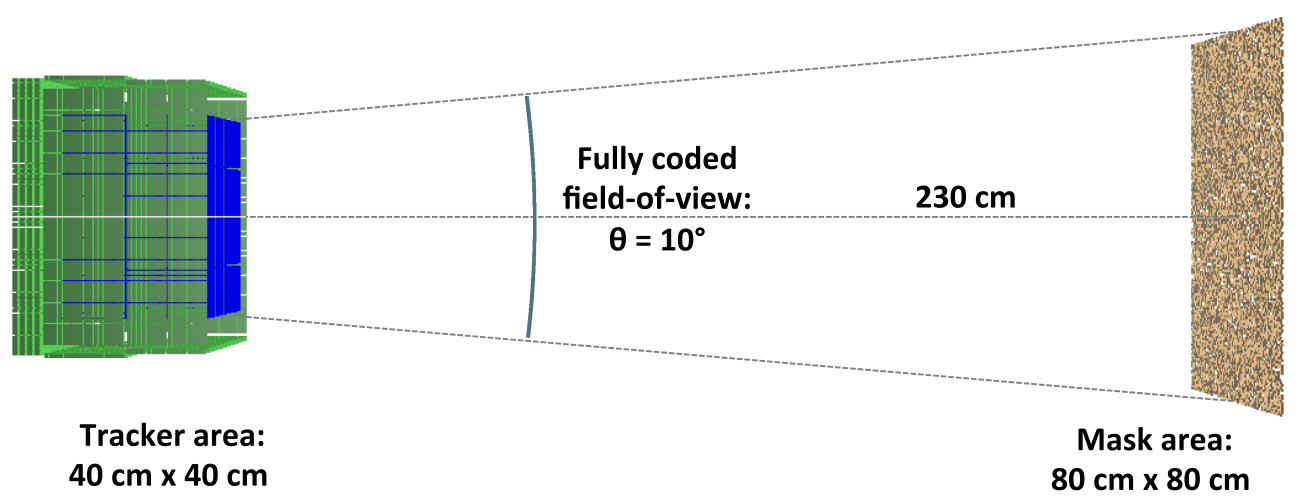

Fig. 2. Compton telescope combined with a tungsten coded mask at a separation distance of $2.3 \mathrm{~m}$, allowing for a fully coded FoV of $10^{\circ}$.
Each silicon wafer has an active area of $10 \times 10 \mathrm{~cm}$ surrounded by a $1.5 \mathrm{~mm}$ guard ring. At the top and bottom of the silicon material are strip electrodes of $0.5 \mathrm{~mm}$ width. The top strips are orthogonal to the bottom strips in order to obtain $x-y$ position information for each interaction. The spatial resolution of the silicon detectors is given by the $0.5 \mathrm{~mm}$ strip pitch, thus is a contributing factor that allows for good angular resolution of the telescope. Several wafer thicknesses were simulated to optimize the angular resolution and effective area at $511 \mathrm{keV}$ before arriving at a thickness of $2 \mathrm{~mm}$.

The tracker consists of $4 \times 4$ wafers, yielding a total geometric surface area of $1600 \mathrm{~cm}^{2}$. As with the GRIPS telescope, 64 layers of wafers were used (1024 total Si detectors) with the vertical spacing between layers set to $0.5 \mathrm{~cm}$ in order to provide a high Compton efficiency over a wide FoV. This yields a total tracker depth of $\sim 33 \mathrm{~cm}$. The total mass of silicon in the tracker is $\sim 50 \mathrm{~kg}$.

\subsection{CdZnTe absorbers}

For this work, the lanthanum bromide calorimeters that were used in the GRIPS design were replaced with $1 \mathrm{~cm}^{3}$ coplanargrid CdZnTe detectors (Luke 1995, 1996; Amman \& Luke 1999). The large-volume ambient-temperature CdZnTe detectors have the advantage of simplified electronics, low power consumption, a high active-to-passive material ratio, and a high average $Z\left(Z_{\text {ave }}=49\right)$, making them a good candidate for a Compton telescope absorption plane. Particularly, the background is minimized because there is very little passive material surrounding the instrument, which is is possible because the detectors have little or no cooling requirements. The $\mathrm{CdZnTe}$ detector response is well understood after successful benchmarking of laboratory measurements with the High Efficiency Multimode Imager (HEMI; Galloway et al. 2011), a combined Compton and coded-aperture instrument developed for nuclear security applications. As a result of detector development during the HEMI campaign, the cubic-centimeter CdZnTe detectors were fabricated with good spectral performance (Amman et al. 2009). The energy resolution of the highest-performing CdZnTe detector was simulated in this case, that is, 1.5\% FWHM at $662 \mathrm{keV}$, as a reasonable approximation of the obtainable energy resolution over a long-term production phase. Although this assumes room temperature operation, it is noted that a $\sim 30 \%$ improvement in energy resolution can be achieved with moderate cooling to $-20^{\circ} \mathrm{C}$ (Amman et al. 2006). A benchmarked time resolution of $0.5 \mu$ s was used in the simulation.

The absorber planes consist of a single layer of 7200 elements surrounding the tracker on four sides and a $52 \times 52$ array of elements four layers deep underneath the tracker (10 816 elements). Each individual element includes front-end electronics and a Lexan housing, as in the HEMI design. The number of layers surrounding the tracker on all five sides was optimized to achieve full absorption of high-energy photons within the sensitive volume of the telescope without having excess detector material. The final model contains $18016 \mathrm{CdZnTe}$ detector elements with a total mass of $\sim 103 \mathrm{~kg}$.

\subsection{Coded mask}

The coded aperture design began with the choice of a mask pattern with a specified ratio of transparent elements to total mask area, that is, the open fraction denoted as $f_{e}$. To obtain the best signal-to-noise ratio, the open fraction of the mask was optimized according to the expected point-source intensity relative to the background intensity (Gunson \& Polychronopulos 1976). It was found that the optimum fraction is $f_{e}=1 / 2$ if the background dominates the source intensity. This result was reviewed under various detection scenarios (In't Zand 1992; Skinner 2008). Considering the real-world application in medium-energy gamma-ray astrophysics, an open fraction of $f_{e}=1 / 2$ is an appropriate choice, as used by the Burst Area Telescope (BAT) on Swift (Barthelmy et al. 2005).

The mask with telescope is shown in Fig. 2. The mask element spacing, the distance from the mask to the Compton telescope, and the geometric area of the mask were designed to provide an angular resolution, $\Theta$, in pointing mode of $0.125^{\circ}$ ( $7.5 \mathrm{arcmin})$ and a $10^{\circ} \mathrm{FoV}$. The mask consists of cubiccentimeter tungsten pixels $(Z=84)$ with a size of $80 \times 80 \times$ $0.5 \mathrm{~cm}$ arranged in a $160 \times 160$ random mask pattern (not optimized). A support structure for the mask is not included in the model. The mask-detector distance is $230 \mathrm{~cm}$. The geometric mask area is $6400 \mathrm{~cm}^{2}$, with a total tungsten mass of $\sim 30 \mathrm{~kg}$.

\subsection{Anti-coincidence shield}

An organic plastic scintillator anti-coincidence (AC) shield surrounds the Compton telescope on all sides (Fig. 1, red). The AC shield allows to veto external background events that are primarily induced by albedo and semi-trapped charged particles, as well as from cosmic rays that are not already reduced by a low-Earth orbit (described in Sect. 6.1). An energy resolution of $10 \mathrm{keV}$ ( $1 \sigma$ Gaussian) and a trigger threshold of $50 \mathrm{keV}$ were used for the shield. The total mass of the plastic scintillator and related components is $\sim 70 \mathrm{~kg}$.

\section{Analysis tools and methods}

The Medium Energy Gamma-ray Astronomy library software package, MEGAlib (Zoglauer et al. 2006) was used for all aspects of the data analysis, including simulations, Compton 
event reconstruction, and both Compton and coded-aperture imaging. MEGAlib is written in $\mathrm{C}++$ and based on ROOT (Brun \& Rademakers 1997). It is an open source software package that was designed for use with gamma-ray detectors, particularly for imaging with Compton telescopes.

Monte Carlo simulations were performed using Cosima (Zoglauer et al. 2009), the simulation toolkit contained in MEGAlib. In order to simulate the performance of the telescope, a realistic geometry was first created using the Geomega package in MEGAlib (Figs. 1 and 2). The geometry specifies the dimensions of each volume, the elemental composition, and density of all passive and active volumes within the detector and surrounding environment. The simulator imports the geometry and converts it into a Geant4 (Agostinelli et al. 2003) format. Cosima then uses the specified source information to simulate particle propagation and interactions with the modeled materials, for example, photoabsorption, Compton and Rayleigh scattering. The resulting Cosima output file contains the simulated interaction information, including the time, type, position, and energy deposition of each interaction. The simulated hits are convolved with the detector response using the detector effects engine contained in MEGAlib.

\subsection{Compton reconstruction}

For Compton reconstruction as applied in this work, coincident hits are merged in the simulation, therefore adjacent hits were clustered together to form Compton events. Compton sequence reconstruction, CSR (Zoglauer 2005), was then applied to the convolved data to determine the sequence of each event, thus finding the initial photon energy and the most likely scattering angle of the incoming photon. It was required in CSR that the first hit occur in the silicon tracker. Nominally, this implies an FoV of $<90^{\circ}$ because of the positioning of the Si tracker inside the $\mathrm{CdZnTe}$ well, but as a result of the passive material surrounding each CdZnTe detector, transmission still occurred at higher incidence angles to allow for the first interaction to take place in the tracker. Therefore the performance was evaluated over a $120^{\circ}$ FoV. Additionally, an Earth horizon cut of $90^{\circ}$ was used to eliminate events that originate at or below the horizon to reduce background events generated from albedo photons.

This list of reconstructed events then underwent imaging and other high-level analysis such as application of event cuts, estimation of angular resolution measurement (ARM), and an assessment of photon interaction locations and energies, using the Mimrec tool in MEGAlib (Zoglauer et al. 2011). Imaging algorithms were used to generate a Compton backprojected image within a user-specified coordinate system. Additionally, a list-mode maximum likelihood expectation maximization (ML-EM) algorithm can be applied to further refine the image (Wilderman et al. 1998).

\subsection{Combined Compton and coded-aperture imaging}

In combined-imaging mode, Compton event reconstruction and imaging is performed first. The region of overlap of the Compton circles identifies the source location with an angular resolution as given by the ARM distribution. The coded-aperture imaging technique is then applied to these selected Compton events, where the first hit can occur in any layer of the silicon tracker. For each reconstructed point of origin, the probability that the photon was photoabsorbed as it passed through the mask is calculated. The result is a shadow pattern projected onto the Compton reconstructed point of origin.
Image - Iteration: 0

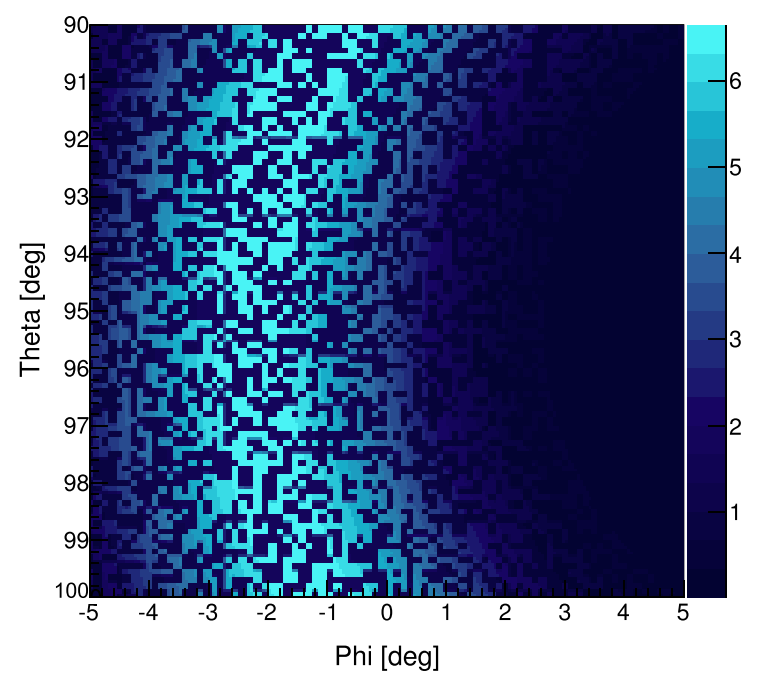

Fig. 3. Reconstructed Compton circle (backprojection) overlaid with the projected mask pattern. The pixellation of the mask within the $10^{\circ} \mathrm{FoV}$ significantly improves the angular resolution.

Table 1. Astrophysical nuclear line emissions and continuum source simulated with the combined-imaging telescope.

\begin{tabular}{ll}
\hline \hline Energy $(\mathrm{keV})$ & Source \\
\hline 200 & Nonspecific \\
511 & $\mathrm{e}^{+} \mathrm{e}^{-}, \beta^{-}$decays, etc. \\
847 & ${ }^{56} \mathrm{Ni} \rightarrow{ }^{56} \mathrm{Co}$ \\
1157 & ${ }^{44} \mathrm{Ti}$ \\
1332 & ${ }^{60} \mathrm{Fe} \rightarrow{ }^{60} \mathrm{Co}$ \\
1809 & ${ }^{26} \mathrm{Al}$ \\
4440 & ${ }^{12} \mathrm{C}^{*}$ molecular de-excitation \\
6130 & ${ }^{16} \mathrm{O}^{*}$ molecular de-excitation \\
$511 \pm 1.25$ & Broadened $\mathrm{e}^{+} \mathrm{e}^{-}$line \\
Continuum & Power-law index 2.17 \\
\hline
\end{tabular}

The strength of adding a coded mask to a Compton telescope is exemplified in Fig. 3, which shows a coded-mask pattern backprojected onto a reconstructed Compton cone from a single event. The pixellation of the pattern limits the point of origin from the width of the ARM distribution to that given by the mask geometry, thus greatly improving the angular resolution. Likewise, the background rejection from the Compton reconstruction eliminates a significant area of the backprojected mask, thus coding noise is not an issue and a greatly improved source localization is obtained compared to coded-aperture mode alone.

\section{Simulated performance}

The instrument was placed in a low-Earth, near-equatorial orbit. A series of monoenergetic far-field line sources from $200 \mathrm{keV}$ to $10 \mathrm{MeV}$ were simulated on-axis, as described in Table 1. A $511 \mathrm{keV}$ source broadened by $\sigma=1.25 \mathrm{keV}$ was also simulated at various incident angles. The broadening is based upon observations of the line from INTEGRAL/SPI (Jean et al. 2006). Additionally, a Crab-like source with a power-law index of 2.17, also observed by SPI (Sizun et al. 2004; Vedrenne et al. 2003), was simulated to predict the sensitivity of the telescope to continuum sources. 

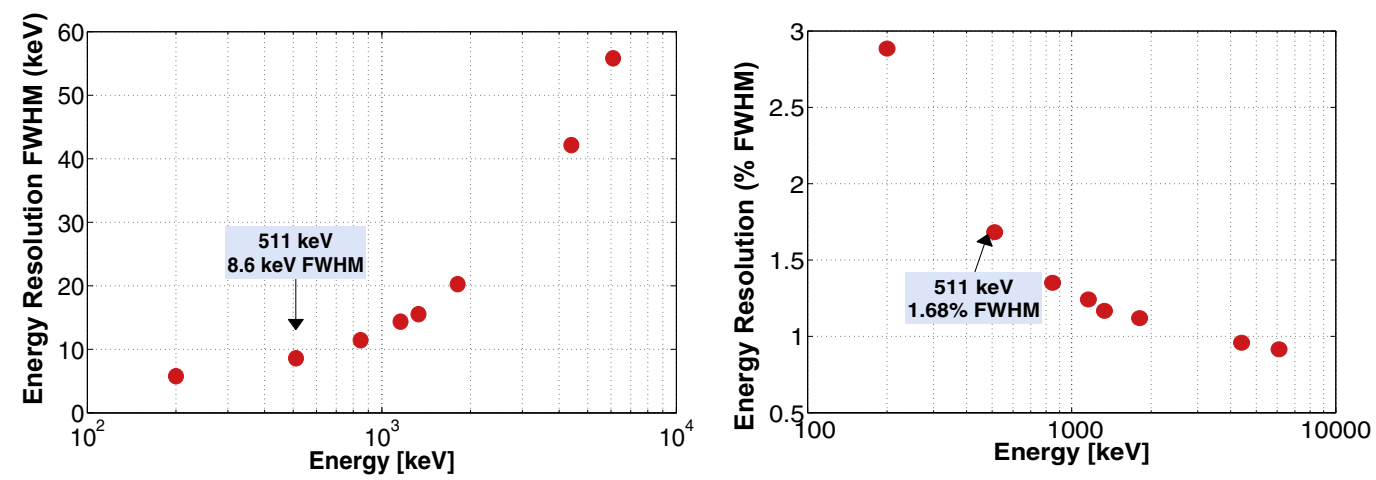

Fig. 4. Energy resolution, including multiple-site events, as a function of energy in terms of the FWHM, left, and percent, right.
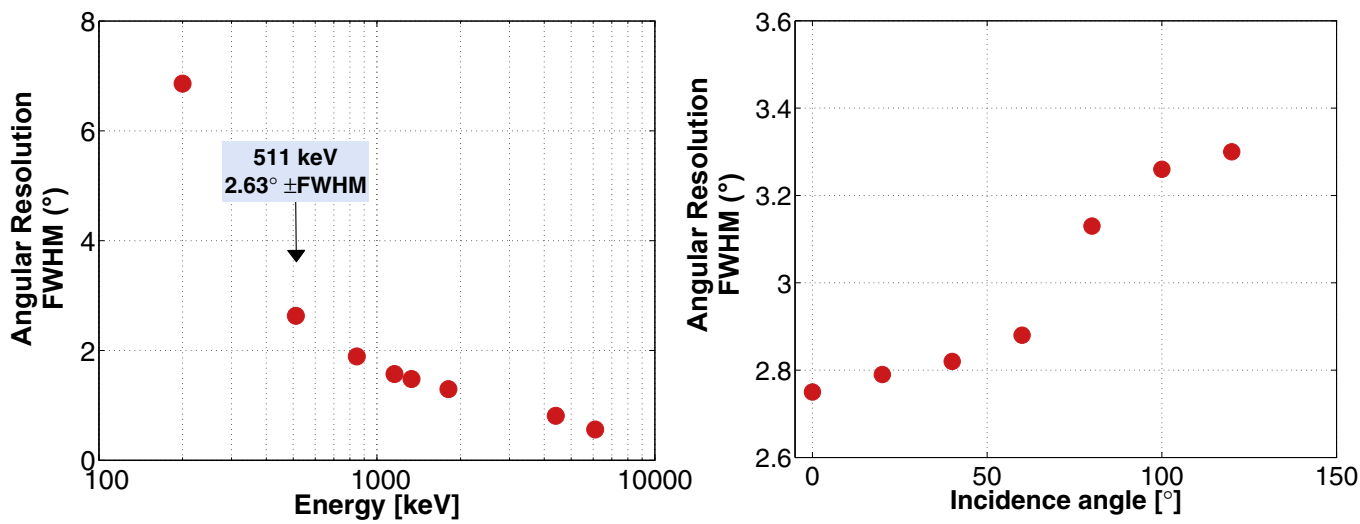

Fig. 5. ARM (Compton mode) as a function of energy, left, and incidence angle, right, for a $511 \mathrm{keV}$ broadened line source, right.

\subsection{Energy resolution}

The telescope energy resolution depends upon the energy resolutions of both the silicon tracker and the CdZnTe absorbers. For each hit, the energy resolution adds in quadrature to achieve the total energy resolution for each event. Equation (1) describes the total energy resolution for an $n$-site event, where $E_{i}$ is the energy resolution of the detector where hit $i$ occurred,

$\Delta E_{\mathrm{tot}}=\sqrt{\sum_{i=1}^{n} \Delta E_{i}^{2}}$.

Because most of the Compton interactions occur in the tracker, the good energy resolution of the Si DSSD detectors is reflected in the overall energy resolution of the telescope.

Using the performance parameters described in the previous section, the simulated photopeak energy resolution for the telescope is shown in Fig. 4 as a function of energy in terms of the FWHM, left, and percent, right. This estimation includes multiple-site events. The overall energy resolution of the telescope corresponds to $1.68 \%$ FWHM at $511 \mathrm{keV}$.

\subsection{Angular resolution}

For Compton events, the ARM is defined as the angular distance between a known source position and the closest reconstructed position on the Compton cone, that is, the ARM is the width of the Compton cone, and thus it represents the power to resolve point sources (Schoenfelder et al. 1993). It is a function of energy resolution, position resolution, and Doppler broadening. Figure 5 shows the ARM as a function of energy, left, and incidence angle for a $511 \mathrm{keV}$ broadened line source, right. $\mathrm{A} \pm 3 \sigma$ photopeak energy window was used to determine the ARM.

The degradation in the ARM at higher incidence angles is due to geometric effects. Because a photon is more likely to scatter into a neighboring detector rather than between planes at higher incidence angles, the average distance between the first two interactions as seen from simulations is shorter: $9.9 \mathrm{~cm}$ at $120^{\circ}$ vs. $13.2 \mathrm{~cm}$ for on-axis photons. The limiting factor in the achievable angular resolution for the telescope in Compton mode is the position resolution from the $0.5 \mathrm{~mm}$ strip pitch of the silicon detectors and particularly the cubic-centimeter voxel size of the CdZnTe detectors.

The angular resolution of a coded-mask instrument is the smallest angular distance at which two point-like sources can be resolved. Conservatively, this distance is taken to be 1.26 times the FWHM of the detected point spread function (Dean \& Byard 1991). The angular resolution is determined by the instrument geometry, as shown in Eq. (2) (Skinner 2008), where $m$ is the horizontal spacing between the mask pixels, $d$ is the position resolution of the detector elements, and $L$ is the distance from the mask to the detection plane,

$\delta \theta^{2}=(m / L)^{2}+(d / L)^{2}$.

Given that the first Compton event occurs in the DSSD, the pixel spacing is much larger than the detector spatial resolution, $d \ll m$, thus the angular resolution can be approximated by Eq. (3),

$\Theta=\arctan (m / L)$.

The mask-detector distance is a trade-off between Compton efficiency, as decreasing the separation distance between planes 
yields more Compton scatter events, and angular resolution, as larger separation distances yield better angular resolution in both Compton and coded-mask mode. Furthermore, at larger standoff distances, the background is minimized, since gamma rays that originate from activation in mask materials are less likely to hit the detection plane. A separation distance between the mask and the top plane of the tracker of $L=2.3 \mathrm{~m}$ and a pixel spacing of $m=0.5 \mathrm{~cm}$ were chosen to yield an angular resolution of $0.125^{\circ}$ within a $10^{\circ} \mathrm{FoV}$ and at all energies. This is roughly a factor of 20 improvement at $511 \mathrm{keV}$ over the achievable angular resolution in Compton mode.

The point source location accuracy (PSLA), defined as the degree of uncertainty to which a point source can be located, is given by Eq. (4) in the case where $d \ll m$ (Stephen 1991), where $\sigma$ is the significance of detection,

$\operatorname{PSLA}(1 \sigma)=\arctan \left(\frac{m}{L}\right) * \frac{1}{\sigma}$.

Thus a $3 \sigma$ detection yields a position uncertainty of $\pm 0.04^{\circ}$.

For image reconstruction, the first hit can occur in any detection plane of the tracker, thus the angular resolution improves slightly as a function of interaction depth and is also energy dependent. However, at $511 \mathrm{keV}$, the probability that the first Compton scatter occurs in the top half of the tracker is $>70 \%$. In this case the improvement in angular resolution is less than $\sim 4 \%$, thus is considered negligible for this study.

\subsection{Effective area}

Because not all of the photons that are incident upon the geometric area of a detector interact to produce counts or real events in the detector, the effective area is a reduction from the actual geometric area of a detector. This reduction is due to the intrinsic detection efficiency and the event cuts required for Compton reconstruction. The event cuts include an energy window of $\pm 1.4 \sigma$ around each line energy, an ARM cut of $\pm 3 \sigma$, and an Earth horizon cut. The effective area as a function of energy and of incidence angle is shown in Fig. 6; the effective area is $182.7 \pm 0.4 \mathrm{~cm}^{2}$ for the Compton telescope without the mask at $511 \mathrm{keV}$, for instance. As the characterization is based on simulation, the uncertainties are statistical and come from the number of simulated events. No mask was included in these simulations.

\section{Sensitivity estimates}

The sensitivity of a Compton telescope can be determined based upon the effective area estimates and a model of the expected background flux. There are several challenges to achieving a high sensitivity in a Compton telescope. The flux from astrophysical gamma-ray sources is typically low, especially compared to the background flux from cosmic rays and the Earth's albedo. Additionally, activation of both passive and active components of the telescope, particularly from cosmic-ray interactions as well as charged particles trapped in the Earth's magnetic fields, creates a pervasive source of background that generally either reaches a statistical equilibrium or continues to increase over time. In addition to the low flux from target gamma-ray sources, the total interaction crosssection reaches a minimum in the Compton regime. Therefore good background reduction techniques are essential for obtaining high sensitivity and can be achieved through a combination
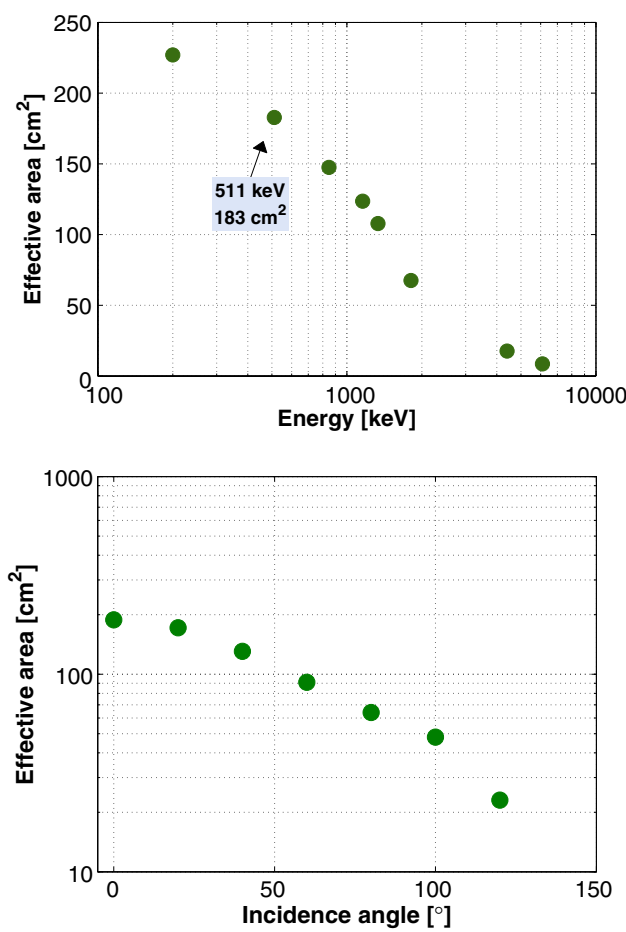

Fig. 6. Effective area as a function of energy, top, and as a function of incidence angle for a broadened $511 \mathrm{keV}$ source, bottom.

of a good telescope design, good energy and angular resolution, discrimination using CSR, and through careful event selections.

Additional challenges are present for achieving high sensitivity in coded-aperture mode. Because source localization is deduced by projecting the spatial locations of the detected counts onto the sky, the background counts are also backprojected along with source counts, thus complicating the ability to discriminate source from background in mask mode. An effective deconvolution algorithm or the ability to operate the telescope in on/off mode to distinguish source from background can allow for better discrimination. Although these techniques are most effective under the assumption of a uniform background distribution, they can be applied when long exposure times allow for accurate background characterization.

In the case of a passive coded mask, events are lost due to absorption in the mask, thus reducing the already limited number of source photons. Additionally, the mask in most cases is neither perfectly transparent nor perfectly opaque. Thus the loss is a function of the filled area of the mask, the energy-dependent transmission through the mask, and absorption or scattering in materials that surround the sensitive detector volume, such as support structures or dead layers in the detectors.

In addition to these nonidealities, the sensitivity and effectiveness of mask-mode observation also depends on the angle of incidence of the source. With regard to this design, only a partially coded FoV can be obtained for all incident sources that are outside of the $10^{\circ}$ pointing mode.

\subsection{Background model}

Because gamma-rays are absorbed by the Earth's atmosphere, observations of astrophysical sources require placing the telescope at balloon altitudes or in space. The expected background is hugely dependent upon the choice of orbit for the 


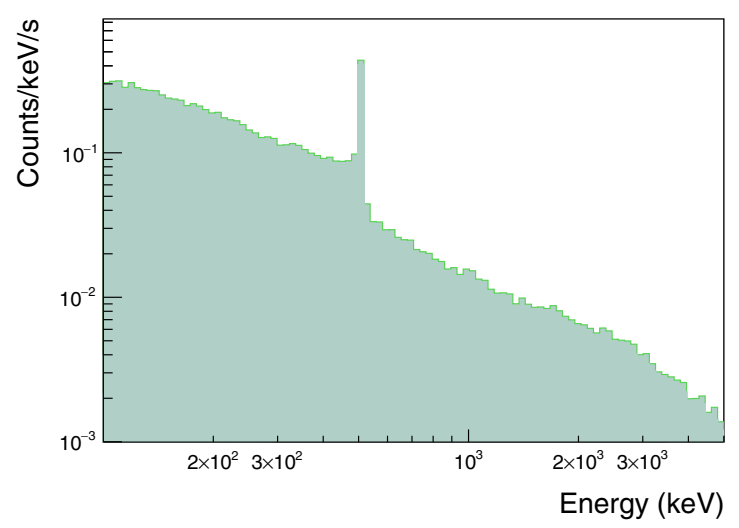

Fig. 7. Reconstructed background after sensitivity optimized event cuts for a $575 \mathrm{~km}$ orbit with a $6^{\circ}$ inclination. Simulated background includes cosmic and albedo photons, leptons, and hadronic components from both prompt interactions and activation.

satellite. In this case, a near-equatorial, low-altitude orbit was chosen. This orbit would minimize exposure to cosmic rays and to high-energy charged particles in the South Atlantic Anomaly. A $575 \mathrm{~km}$ orbit with a $6^{\circ}$ inclination has been extensively studied for the NuSTAR mission (NuSTAR collaboration), thus the NuSTAR pre-flight background model was used for this study.

The gamma-ray background, shown in Fig. 7, consists of extragalactic X-rays and gamma rays, annihilation photons, albedo photons up to several $\mathrm{MeV}$, electrons, positrons, protons, and neutrons. Activation within passive and active volumes of the instrument from hadronic interactions were included in the simulation. The particle distributions were isotropic except for the observed enhancement of albedo photons at zenith angles of $120^{\circ}$. The Compton-reconstructed background components were simulated and then reconstructed using the BackgroundMixer program in MEGAlib. The multiple components show that the $511 \mathrm{keV}$ background is excessive, thus making high sensitivity of true sources at this energy very challenging.

\subsection{Sensitivity in Compton mode}

The telescope sensitivity is a description of the minimum source flux that can be measured by the instrument within a given significance above background. At the sensitivity limit, the number of detectable source counts, $N_{s}$, is given by the minimum flux, $F_{\min }$, the effective area, $A_{\text {eff }}$, and the observation time, $T_{\mathrm{obs}}$, according to Eq. (5),

$N_{s}=F_{\text {min }} A_{\text {eff }} T_{\text {obs }}$.

The number of source counts that satisfies the minimum detectable flux is dependent upon the signal-to-noise ratio, $\sigma$, as shown in Eq. (6), where $\Delta M_{s}$ is the uncertainty in the measurement,

$\sigma=\frac{N_{s}}{\Delta M_{s}}$

Under the condition of Gaussian or Poisson statistics, the measurement uncertainty is described by Eq. (7), which considers the uncertainty in measuring both the source counts, $\Delta S$, and the background counts, $\Delta B$, where $N_{b}$ is the number of detected background counts,

$\Delta M_{s}=\sqrt{\Delta S^{2}+\Delta B^{2}}=\sqrt{N_{s}+N_{b}}$.
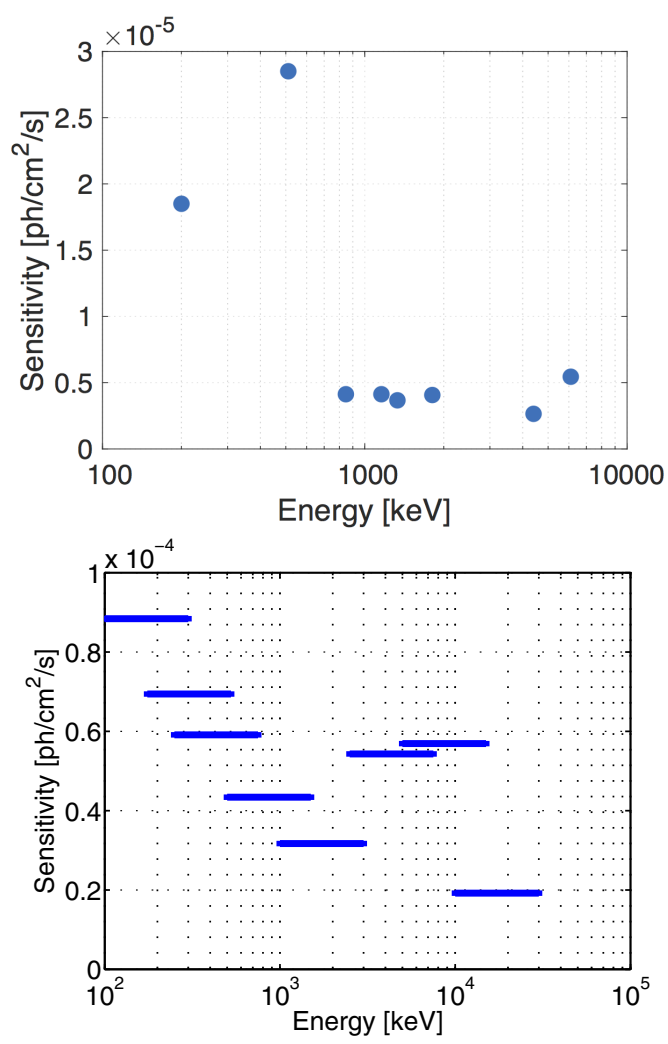

Fig. 8. 3 $\sigma$ sensitivity as a function of line source energy, top, and continuum sensitivity for a simulated Crab-like source, bottom, over an observation time of $1 \mathrm{Ms}$.

An expression for the minimum flux can be derived using Eqs. (5) and (6). In this case, $\sigma$ is a factor of $n$, defined as the detection significance of the source above background, typically $3 \sigma$ or $5 \sigma$. The resulting equation is shown in Eq. (8) (Jacobson et al. 1975),

$F_{\min }(E)=\frac{n^{2}+n \sqrt{n^{2}+4 N_{b}}}{2 A_{\mathrm{eff}} T_{\mathrm{obs}}}$.

Figure 8 shows the calculated $3 \sigma$ sensitivities with optimized event cuts as a function of energy for monoenergetic narrowline sources, top, and the continuum sensitivity for a simulated Crab-like source with a power-law index of 2.17 , bottom. The observation time was $1 \mathrm{Ms}$ in pointing mode. For scanning the whole FoV, a $511 \mathrm{keV}$ broadened line source was simulated separately, resulting in an all-sky sensitivity of $3.6 \times 10^{-6} \mathrm{ph} \mathrm{cm}^{-2} \mathrm{~s}^{-1}$ over a two-year observation time. Simulations for the calculated sensitivities did not include the coded mask.

\subsection{Sensitivity in combined Compton and coded-mask mode}

To evaluate the sensitivity of a coded-aperture instrument, several factors that can contribute to image non-uniformity and that are also relevant for Compton-coded-mask (CCM) imaging mode need to be considered (Skinner 2008). For this study, no support structure existed for the mask, the simulated detector response was identical for each element, the observation times allowed for Gaussian statistics, and the modeled background was spatially uniform. The sensitivity in mask mode was evaluated at or near the center of the FoV, thus considering only fully coded detection scenarios and neglecting nonidealities that are due to the thickness of the mask elements. Furthermore, the mask sensitivity was evaluated only when combined with the Compton 


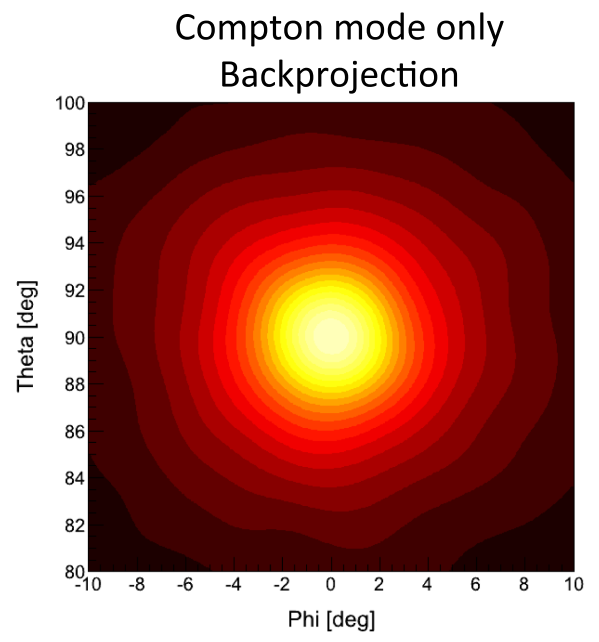

Combined Compton-coded

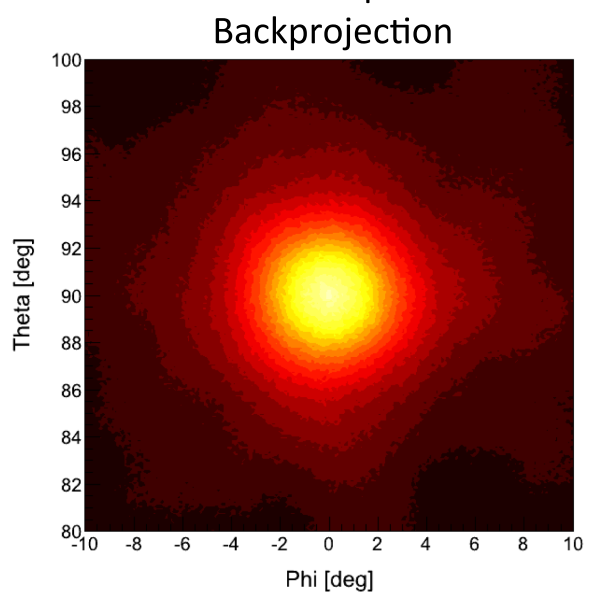

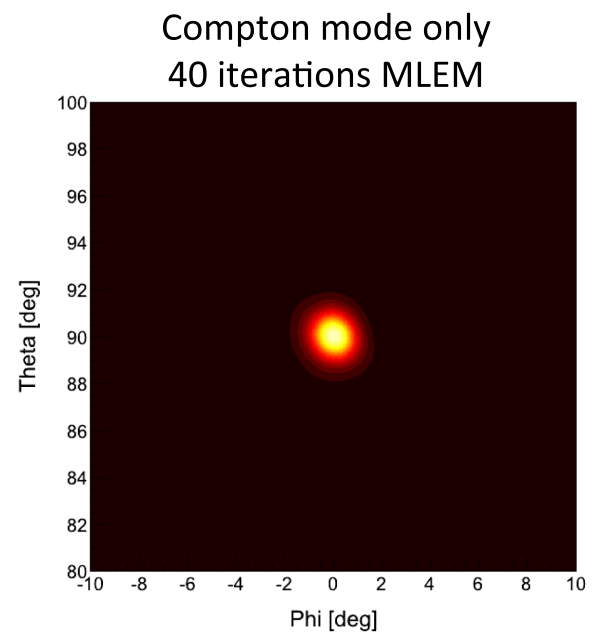

Combined Compton-coded

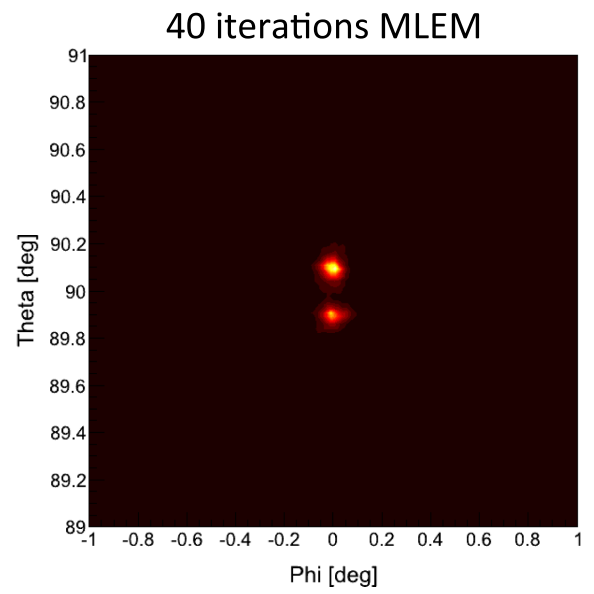

Fig. 9. Imaging of two sources separated by $0.2^{\circ}$. Top left: back projection in Compton mode alone. Bottom left: back projection with combined Compton-coded mask mode. Top right: deconvolved image after 40 iterations in Compton mode alone. Bottom right: combined Compton and codedmask mode image after 40 iterations. Note the change in scale between the bottom two images. telescope. In this case, source photons that pass through the mask unimpeded due to the energy-dependent opacity of the mask elements are compensated for by their deposition in the detection planes and subsequent Compton reconstruction. This allows for background reduction before the mask elements are used to enhance the angular resolution.

As consequence, the sensitivity in CCM mode can be calculated using the same equation as in Compton mode, as the background events contributing to the CCM sensitivity are the same events that contribute to the source in Compton mode. Under the above scenario and considering a mask with open fraction $f=1 / 2$, the number of source counts (i.e., the effective area) is reduced by half, thus requiring twice the minimum flux as compared to Compton-mode alone. This approximation is valid within the fully coded FoV, assuming only single sources are present, and considering a background-dominated case where detection occurs at the sensitivity limit.

\section{Image reconstruction}

Figure 9 shows a demonstration of the multimode imaging capabilities of the telescope. Simulations of two $511 \mathrm{keV}$ point sources separated by $0.2^{\circ}$ were performed. A list-mode maximum-likelihood expectation-maximization algorithm was used for the deconvolved images. In the top two images, only reconstructed Compton events were used. The two sources cannot be separated either before or after deconvolution. The image reconstruction in the bottom two images takes into account the absorption probabilities of the initial photons through the coded mask. The image before the deconvolution shows some additional noise that is due to the overlapping coded-mask patterns superimposed onto the Compton imaging space. However, the deconvolved image clearly shows that the two sources can be separated.

\section{Comparison with other space instruments}

To assess the capabilities of the CdZnTe-based combined imager as a future telescope, Table 2 shows a comparison of performance parameters for a broadened $511 \mathrm{keV}$ source of the instrument with other medium-energy gamma-ray telescopes, such as the coded-mask telescope INTEGRAL/SPI and the proposed e-ASTROGAM Compton telescope (De Angelis et al. 2017). The latter is based upon a silicon tracker and a thallium-activated cesium iodide calorimeter. The table shows that for nuclear line spectroscopy, the germanium-based SPI instrument significantly exceeds the performance of the CdZnTe-based telescope in terms of energy resolution. However, the angular resolution without the mask is comparable to the other two instruments. A great improvement at lower energies is seen in angular resolution with the use of the mask. Theoretically, a mask can be added to any future Compton telescope in order to achieve a high angular resolution at energies below $\sim 0.5 \mathrm{MeV}$, depending upon the mask thickness and pixel material. 
Table 2. Telescope performance comparison of a broadened line source at $511 \pm 1.3 \mathrm{keV}$ for a $10^{6}$ second observation time.

\begin{tabular}{|c|c|c|c|}
\hline & This work & INTEGRAL/SPI & e-ASTROGAM \\
\hline $\begin{array}{l}\text { Energy resolution } \\
\text { (\% FWHM) }\end{array}$ & 1.8 & 0.38 & 3 \\
\hline $\begin{array}{l}\text { Angular resolution } \\
\left({ }^{\circ} \mathrm{FWHM}\right)\end{array}$ & $\begin{array}{l}2.1^{\circ} \text { Compton } \\
<0.13^{\circ} \text { with mask }\end{array}$ & $2.5^{\circ}$ & $2.5^{\circ}$ \\
\hline $\begin{array}{l}\text { Effective area } \\
\left(\mathrm{cm}^{2}\right)\end{array}$ & $\begin{array}{l}143 \\
\sim 72 \text { with mask }\end{array}$ & $\sim 100$ & 446 \\
\hline $\begin{array}{l}3 \sigma \text { sensitivity on-axis } \\
\left(\mathrm{ph} \mathrm{cm}^{-2} \mathrm{~s}^{-1}\right)\end{array}$ & $\begin{array}{l}2.9 \times 10^{-5} \\
5.8 \times 10^{-5} \text { with mask }\end{array}$ & $5.2 \times 10^{-5}$ & $4.1 \times 10^{-6}$ \\
\hline
\end{tabular}

Notes. For this study and for e-ASTROGAM, the angular resolution was optimized for sensitivity, and reconstruction was performed without electron tracking.

In terms of sensitivity, the CdZnTe-based combined imager is a good candidate for a next-generation telescope. In both imaging modalities, it exceeds the sensitivity of SPI, and within the fully coded FoV, is comparable to that of e-ASTROGAM at $511 \mathrm{keV}$. However, a direct comparison is difficult because the geometric area of e-ASTROGAM and consequently its effective area are much larger than for this telescope. For a direct comparison, the instrument would need to be scaled and other factors would also need to be considered, such as cost and detector availability.

\section{Summary}

Based upon laboratory measurements and benchmarked simulations of the HEMI CdZnTe detectors, the combined imager studied in this work can achieve energy resolutions of $1.68 \%$ FWHM at $511 \mathrm{keV}$ and $1.11 \%$ at $1809 \mathrm{keV}$, on-axis angular resolutions in Compton mode of $2.63^{\circ} \pm F W H M$ at $511 \mathrm{keV}$ and $1.30^{\circ} \pm F W H M$ at $1809 \mathrm{keV}$, and it is capable of resolving sources to at least $0.2^{\circ}$ at lower energies, for example, $511 \mathrm{keV}$, with the use of a coded mask. An initial assessment of the instrument yields an effective area of $183 \mathrm{~cm}^{2}$ at $511 \mathrm{keV}$ and an anticipated all-sky sensitivity of $3.6 \times 10^{-6}$ photons $\mathrm{cm}^{-2} \mathrm{~s}^{-1}$ for a broadened $511 \mathrm{keV}$ source over a two-year observation time. Additionally, combining a coded mask with a Compton imager to improve point-source localization for positron detection has been demonstrated.

These capabilities meet several scientific objectives in medium-energy gamma-ray astronomy, such as achieving the sensitivity and accuracy required for nuclear line studies, and in particular, allowing for improved observations of the spatial distribution of Galactic $511 \mathrm{keV}$ emission. Although a future telescope with better energy resolution would significantly improve the sensitivity to line emission, the achievable sensitivity from this design would serve well as a telescope for an all-sky survey. A CdZnTe detector array, in particular, is a good candidate as an absorption plane behind a tracker, as demonstrated, or as an absorbing focal plane behind a wave optics telescope, such as in a Laue lens instrument.
Although this study focused primarily on applying the HEMI detector technology and multimode concept to a space mission, improvements to the simulated performance are anticipated with the use of the silicon tracker in its full capacity. The tracker is capable of reconstructing the direction of the recoil electron for incident photon energies above $\sim 2 \mathrm{MeV}$. This reduces the reconstructed Compton circle to an arc, as many incident directions can be eliminated by tracking the recoil electron. Additionally, for energies higher than $5-10 \mathrm{MeV}$, pair production processes dominate in silicon, thus electron-positron tracking can be used to determine the initial photon direction through conservation of momentum.

\section{References}

Abbott, B. P., Abbott, R., Abbott, T. D., et al. 2017, ApJ, 848, L13

Agostinelli, S., Allison, Amako, K., et al. 2003, Nucl. Instr. Meth. A, 506, 250

Ajello, M., Costamante, L., Sambruna, R. M., et al. 2009, ApJ, 699, 603

Amman, M., \& Luke, P. N. 1999, IEEE Trans. Nucl. Sci., 46, 3

Amman, M. S., Lee, J., \& Luke, P. N. 2006, IEEE Trans. Nucl. Sci., 53, 3035

Amman, M., Lee, J. S., Luke, P. N., et al. 2009, IEEE Trans. Nucl. Sci., 56, 795

Bandstra, M. S., Bellm, E. C., Boggs, S. E., et al. 2011, ApJ, 738, 1

Barthelmy, S. D., Barbier, L. M., Cummings, J. R., et al. 2005, Space Sci. Rev., 120,143

Boddy, K. K., \& Kumar, J. 2015, Phys. Rev. D, 92, 023533

Bouchet, L., Roques, J. P., \& Jourdain, E. 2010, ApJ, 720, 1772

Brun, R., \& Rademakers, F. 1997, Nucl. Instr. Meth. A, 389, 1

Dean, A. J., \& Byard, K. 1991, Adv. Space Res., 11, 8

De Angelis, A., Tatischeff, V., Tavani, M., et al. 2017, Exp. Astron., 44, 25

Forot, M., Laurent, P., Lebrun, F., \& Limousin, O. 2007, ApJ, 668, 1259

Galloway, M., Zoglauer, A., Amman, M., et al. 2011, Nucl. Instr. Meth. A, 652, 641

Greiner, J., Iyudin, A., Kanbach, G., et al. 2009, Exp. Astron., 23, 1

Gunson, J., \& Polychronopulos, B. 1976, MNRAS, 177, 485

In't Zand, J. J. M. 1992, PhD Thesis, University of Utrecht, The Netherlands

Jacobson, A. S., Bishop, R. J., Culp, G. W., et al. 1975, Nucl. Instr. Meth., 127, 115

Jean, P., Knödlseder, J., Gillard, W., et al. 2006, A\&A, 445, 579

Kierans, C. A., Boggs, S. E., Chiu, J.-L., et al. 2017, ArXiv e-prints [arXiv: 1701.05558]

Luke, P. N. 1995, IEEE Trans. Nucl. Sci., 42, 4

Luke, P. N. 1996, Nucl. Instr. Meth. A, 380

Oberlack, U., Bennett, K., Bloemen, H., et al. 1996, A\&AS, 120, 311

Padovani, P., Alexander, D. M., Assef, R. J., et al. 2017, A\&ARv, 25, 2

Phillips, M. M. 1993, ApJ, 413, L105

Prantzos, N., Boehm, C., Bykov, A. M., et al. 2011, Rev. Mod. Phys., 83, 1001

Schoenfelder, V., Aarts, H., Bennett, K., et al. 1993, ApJS, 86, 657

Sizun, P., Shrader, C. R., Attié, D., et al. 2004, Proc. of the 5th INTEGRAL Workshop on the INTEGRAL Universe, eds. V. Schönfelder, G. Lichti, \& C. Winkler, ESA SP, 552, 815

Skinner, G. K. 2008, Appl. Opt., 47, 2739

Stephen, J. B. 1991, Adv. Space. Res., 11, 8

Vedrenne, G., Roques, J.-P., Schönfelder, V., et al. 2003, A\&A, 411, L63

Wilderman, S. J., Rogers, W. L., Knoll, G., et al. 1998, IEEE Trans. Nucl. Sci., 45, 957

Zoglauer, A. 2005, Doctoral Thesis, TU Munich, Germany

Zoglauer, A., \& Kanbach, G. 2003, Proc. SPIE, 4851, 1302

Zoglauer, A., Andritschke, R., \& Schopper, F. 2006, New Astron. Rev., 50, 7

Zoglauer, A., Andritschke, R., Boggs, S. E., et al. 2008, New Astron. Rev., 52, 431

Zoglauer, A., Weidenspointner, G., Galloway, M., Boggs, S. E., \& Wunderer, C. B. 2009, in IEEE Nucl. Sci. Symp. Conf. Rec., 2053

Zoglauer, A., Boggs, S. E., Galloway, M., et al. 2011, Nucl. Instr. Meth. A, 652, 568 